\title{
The Role of Transcription Factors in Cancer Angiogenesis and Metastasis
}

ISSN: 2637-773X

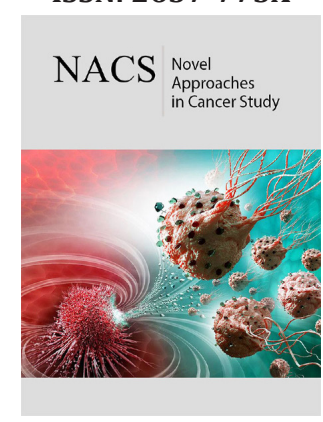

*Corresponding author: Yi Lu, Department of Pathology and Laboratory Medicine, University of Tennessee Health Science Center, Cancer Research Building, 19 South Manassas Street, Memphis, TN 38163, USA, Email: ylu@uthsc.edu

Submission: 温 August 27, 2020

Published: 制September 22, 2020

Volume 5 - Issue 2

How to cite this article: Anming Gu, Jie Wang, Chikezie O Madu, Yi Lu. The Role of Transcription Factors in Cancer Angiogenesis and Metastasis. Nov Appro in Can Study. 5(2). NACS.000609. 2020. DOI: $10.31031 /$ NACS.2020.05.000609

Copyright@ Yi Lu, This article is distributed under the terms of the Creative Commons Attribution 4.0 International License, which permits unrestricted use and redistribution provided that the original author and source are credited.

\author{
Anming Gu${ }^{1}$, Jie Wang ${ }^{1}$, Chikezie 0 Madu $^{2}$ and Yi Lu ${ }^{3 *}$ \\ ${ }^{1}$ Departments of Biology and Advanced Placement Biology, White Station High School, \\ Memphis, TN 38117, USA, gu.anming106@gmail.com, jiewang2002@gmail.com \\ ${ }^{2}$ Departments of Biology and Advanced Placement Biology, White Station High School, \\ Memphis, TN 38117, USA, maduco@scsk12.org \\ ${ }^{3}$ Department of Pathology and Laboratory Medicine, University of Tennessee Health Science \\ Center, Memphis, TN 38163, USA, ylu@uthsc.edu
}

\begin{abstract}
The development of new blood vessels, or angiogenesis, is crucial in cancer progression and metastasis due to its ability to allow the establishment of tumor cells at secondary sites. Because avascular tumors are unable to grow larger or metastasize without sufficient blood supply, avascular tumors are severely restricted in potential. Factors that regulate transcription in cancer angiogenesis and metastasis include those that enhance and inhibit those pathways. Common pathways include the VEGF signaling pathway in angiogenesis and the c-Myb inhibitory transcription factor in metastasis. As a result, targeting these transcription factors may be a valuable approach to improving the prognosis for cancer patients. This review evaluates the role of transcription factors in angiogenesis and metastasis, and the therapeutic potential of targeting these transcription factors.
\end{abstract}

\section{Introduction}

Cancer is a complex pathway that is normally evaded by cells through homeostatic mechanisms. Various genetic and epigenetic alterations may occur along signaling pathways controlling cell growth and division, apoptosis, motility, and fate, causing hyperactivation or inactivation of these pathways. Normally, cells display density-dependent inhibition, only proliferating to a certain density depending on the amount of available growth factors and nutrients. Cancer cells, on the other hand, may either produce their own growth factors, inducing continuous cell division, or have proteins or growth factors that are abnormally regulated. Oncogenic mutations may also affect these signaling pathways, causing gene amplification or dysregulation. Hyperactive pathways may cause excessive cell proliferation by affecting transcription of certain proteins along the pathway or inactivating negative regulators such as $\mathrm{p} 53$, which acts as a tumor suppressor [1].

Cells become malignant once they metastasize and establish secondary tumors throughout the body, making them much more resistant to treatment and therefore much more dangerous [2]. The metastatic cascade occurs when cancer cells detach from the primary tumor and intravasate the blood and lymphatic vessels. To be successful at the secondary site, metastatic cells must establish a microenvironment that allows angiogenesis [3]. Angiogenesis occurs when tissue membranes become injured locally and destroyed. Endothelial cells proliferate and migrate to form a tube, and angiogenic factors facilitate the process, stimulating angiogenesis when the metastatic tissues need nutrients and oxygen. The upregulation of these factors, combined with the downregulation of inhibitors of the pathway, leads to angiogenesis. Without enough blood circulation or vascular support from angiogenesis, tumors become necrotic or apoptotic [4]. Except for cancers of the lungs and liver, carcinomarelated mortality is generally due to metastasis, rarely due to primary carcinoma [4]. Thus, cancer metastasis and angiogenesis are crucial in the progression of most types of cancer. 
Gene expression in cells is regulated by transcription factors that influence RNA polymerase activity, and in cancer cells genes encoding these transcription factors are altered through amplification, deletion, or rearrangement, which causes altered genes. Additionally, transcription factor cofactors, when mutated, may cause tumors [5]. Transcription factors activate or repress the transcription of DNA to RNA by binding to promoter regions on the DNA or directly to the RNA polymerase. These factors are significant in influencing cell division and may be products of oncogenes or tumor suppressor genes [6]. As a result, when this regulation is breached, the cell cycle may progress past checkpoints without the proper transcription factors and cause proliferation and tumorigenesis. Similarly, promotion and inhibition of cancer angiogenesis are regulated by transcription factors. Therefore, understanding the regulation of transcription factors is essential in understanding the role they play in cancer metastasis and angiogenesis [7].

\section{Role of Transcription Factors in Cancer Angiogenesis}

Angiogenesis serves as a foundational step in the metastasis of cancer because cancer cells depend on blood and lymph vessels to adequately supply oxygen and nutrients, as well as to remove waste products. There are several transcription factors that either promote or inhibit angiogenesis [8]. Growth factors, which promote angiogenesis, induce surrounding cells to proliferate and differentiate to become blood vessels, facilitating material exchange. Specifically, in cancer, overexpression of oncogenes due to these growth factors may cause increased expression of genes leading to tumor angiogenesis [9]. The aggressiveness of tumor cells is reflected by the levels of expression of these transcription factors [10]. In a previous study, Muthukkaruppan et al. [11] compared the growth of cancer cells in the iris with blood circulation and the anterior chamber without circulation. Cancer cells in the anterior chamber stopped growing after reaching $1-2 \mathrm{~mm}$ in diameter while those in the iris continued to grow beyond $2 \mathrm{~mm}$ [12]. Furthermore, Holmgren et al. discovered that tumors under angiogenesis suppression a higher rate of necrosis and apoptosis, indicating targeting angiogenesis inhibitors may be a viable approach to obstruct the progression of cancer [13]. Upregulation of growth factors is not sufficient for cancer angiogenesis. Inhibitors of angiogenesis also needs to be downregulated [10]. Neoplastic angiogenesis is essential for the progression of cancer from an avascular stage to a vascularized stage and for tumor growth and metastasis, so pro- and anti-angiogenic factors are explored as potential points for cancer therapy [14]. There are more than a dozen different proteins that have serve as angiogenic factors in tumor development, including positive regulators such as vascular endothelial growth factor (VEGF), basic fibroblast growth factor (bFGF), transforming growth factor (TGF)- $\alpha$, TGF- $\beta$, angiogenin, tumor necrosis factor (TNF)- $\alpha$, placental growth factor, interleukin-8, and negative regulators such as angiostatin, endostatin, and interferon- $\alpha[10,15]$.

Other endogenous angiogenesis inhibitors include proteins, protein fragments, and miRNAs, and they have been continuously discovered throughout recent years. These factors may help downregulate vessel growth by inducing apoptosis, inhibiting migration and formation of tubules, inhibiting growth factors, and binding to receptors which cause blood vessel growth to cease [10]. Two representative proteins include Ismithin 1 (ISM1) and Multimerin 2 (MMRN2) [16]. ISM1, a secreted protein whose biological function has been unknown until recent years, has been found to suppress VEGF induced angiogenesis in mice as well as to induce endothelial cell apoptosis. Data suggests that apoptosis is induced by the protein because it blocks ATP/ADP exchange [16]. Additionally, overexpression of ISM1 has been found to significantly suppress tumor growth in mice by inhibiting angiogenesis [17]. The function of MMRN2 has also remained elusive until it was recently discovered that it inhibits angiogenesis by preventing endothelial cell migration and organization of blood vessels. Furthermore, it only seems to affect abnormal growth, proliferation, or apoptosis in endothelial cells and thus can either limit angiogenesis or maintain a normal state, much like a feedback regulator [16].

\section{Angiogenic Activator VEGF}

Hypoxia is the condition in which a portion of the body is deprived of adequate oxygen supply at the tissue level. Angiogenic activity can be triggered through VEGF by hypoxia via the hypoxiainducible factor- $1 \alpha$ (HIF-1 $\alpha$ ) [10]. HIF-1 $\alpha$ expression initiates following an increase of distance between the capillaries and the growing tumor cells [10]. The VEGF family and their receptors (VEGFR) have received increased attention in recent years. This eight-member family consists of five VEGF glycoproteins (VEGF-A, VEGF-B, VEGF-C, VEGF-D, VEGF-E, VEGF-F) and two placental growth factors (PlGF-1, PlGF-2) (Table 1) [15,18,19]. Of these, VEGF-A is most well-documented, consisting of at least seven homodimeric isoforms due to alternative exon splicing $[15,18]$. More specifically, VEGF121 and VEGF165 comprise the majority of the isoforms and are up regulated in a variety of human tumors $[18,20]$. The VEGF family signals through three tyrosine kinases, VEGFR-1 (Flt-1), VEGFR-2 (KDR), and VEGFR-3 [18,20]. VEGFR-1 and VEGFR-2 mediate most parts of angiogenesis in endothelial cells while VEGFR-3 expression is primarily limited to lymphatic endothelial cells and sometimes to proliferating angiogenic endothelial cells [12].

Table 1: VEGF family members, their Receptors, and gene functions (Adapted from [12]).

\begin{tabular}{|c|c|c|}
\hline VEGF Family Members & Receptors & General Functions \\
\hline VEGF-A & VEGFR-1, VEGFR-2, NAP-1, NAP-2 & $\begin{array}{c}\text { Angiogenesis (NRP-1, NRP-2 function as VEGF } \\
\text { coreceptors), lymphangiogenesis (NRP-2), tumor } \\
\text { cell survival, proliferation, chemotaxis (NRP-1) }\end{array}$ \\
\hline VEGF-B & VEGFR-1 & Angiogenesis \\
\hline
\end{tabular}




\begin{tabular}{|c|c|c|}
\hline VEGF-C & VEGFR-3, NAP-2 & Lymphangiogenesis \\
\hline VEGF-D & VEGFR-3 & Lymphangiogenesis \\
\hline VEGFE & VEGFR-2 & Angiogenesis, lymphangiogenesis \\
\hline PlGF-1 & VEGFR-1 & Angiogenesis \\
\hline PlGF-2 & VEGFR-1 & Angiogenesis \\
\hline
\end{tabular}

It has been shown that each isoform of VEGF has specificity to the type of receptor, depending on the role of each factor. VEGF-A, also known as VEGF/vascular permeability factor (VPF), primarily exhibits angiogenic effects through binding to VEGFR-1 and VEGFR-2 $[12,18,20]$. VEGF-A has a role in endothelial cell proliferation, survival, and microvascular permeability [12]. VEGF-B and the two PGFs utilize VEGFR-1 as their receptor. VEGF-B expression occurs mainly in skeletal muscles, heart muscles, and vascular cells, but its biological function is currently unclear [10]. VEGF-C and VEGF-D primarily exhibit lymphangiogenesis through binding to VEGFR-3, but they may also exhibit vascular angiogenesis through binding to VEGFR-2 [12]. VEGF-E shares approximately $25 \%$ of the same amino acids as VEGF-A, so it also binds to VEGF-2 but not VEGF1 [18]. VEGF-E is encoded by the Orf virus, and it seems to promote endothelial cell growth [10].

VEGF plays significant signaling roles in major areas of the body, and it is highly expressed in malignant tumors, often associated with angiogenesis. VEGF is involved in enhanced endothelial cell proliferation and survival, increased permeability of existing vessels, and enhanced rate of metastasis [18]. VEGF also has several functions that are not related to vascular processes. Its paracrine and autocrine expression and signaling are well documented in cancer cells. It directly influences cancer invasion and migration, and it can induce apoptosis in cells with reduced VEGF expression [18]. VEGF is able to activate endothelial cells which produce substances that break down the extracellular matrix, allowing cell division and migration [18].

Many regulators are involved in VEGF expression, including hypoxia, growth factors and cytokines, oncogenes and tumor suppressor genes, glycosaminoglycans, and miRNA [15]. The hypoxia-inducible factor (HIF) complex increases the rate of transcription of genes such as VEGF in cells with hypoxia [21]. Hypoxia occurs when HIF prolyl hydroxylases detect that available oxygen levels in tissue is below $5 \%$, causing the expression of HIF1 , which is a heterodimeric transcription factor that controls many oxygen-sensitive genes [21,22]. HIF- $1 \alpha$ and HIF- $2 \alpha$ largely have specificity in the genes they activate. HIF-1 $\alpha$ primarily activates genes involving glycolysis and apoptosis while HIF- $2 \alpha$ primarily activates genes involving proliferation and de-differentiation [22]. However, these two subunits both have a role in angiogenesis, invasion, and metastasis [23]. Another important regulator of angiogenesis is glycosaminoglycans (GAG). GAG derivatives have been found to bind to VEGF165, imparing the interaction of it to VEGFR [24]. Tissue inhibitor of metalloproteinase-3 (TIMP-3) is a protein that acts as a competitive inhibitor of angiogenesis by targeting VEGFR-2 [25]. Although it is known that glycosaminoglycans are involved in the interaction of VEGF-2 and TIMP-3, the mechanism remains unclear [25].

MicroRNAs (miRNAs) have recently been increasingly studied due to their involvement in cancer. Several miRs have been shown to modulate angiogenesis by targeting targeting VEGF expression, including miR-148a/b-3p, miR-338-3p, and miR-342-5p [2426]. $\mathrm{miR}-148 \mathrm{a} / \mathrm{b}-3 \mathrm{p}$ regulates the expression of neuropilin-1 (NRP1), which is a transmembrane glycoprotein that serves as the coreceptor for VEGFR-2 [24]. Therefore, the upregulation of miR$148 \mathrm{a} / \mathrm{b}-3 \mathrm{p}$ inhibits the activation of VEGFR-2 and limits angiogenic activity [24]. miR-338-3p also modulates VEGF expression via targeting NRP1 [25]. This miRNA is predicted to bind to the 3'-UTR of NRP1 mRNA, through which it inhibits the activation of VEGF [25]. Similarly, miR-342-5p also limits angiogenic activity, though through a different pathway. The upregulation of miR-342-5p has been shown to reduce VEGF-induced Akt phosphorylation, which decreases the rate of angiogenesis [26].

\section{Angiogenic Inhibitors Angiostatin}

Although angiogenic activators such as VEGF promote angiogenesis, there are also angiogenic inhibitors that are equally important. A balance between pro-angiogenic growth factors and anti-angiogenic growth factors is essential for proper regulation of angiogenesis (Figure 1) [27]. Angiostatin and endostatin are among the first angiogenic inhibitors that were identified [28]. Many recent studies in anti-cancer therapy have focused on the extracellular matrix protein angiostatin. Angiostatin consists of three to five kringle domains surrounded by a $38 \mathrm{kDa}$ fragment from the protein plasmin, which is a fragment of plasminogen [29,30]. Kringle domains fold into large loops stabilized by 3-disulfide linkages. Proper folding of kringle domains is vital for angiostatin to exhert its role as an angiogenic inhibitor [28]. There are several structural variations, based on the combination of kringle domains: K1-3, K1-4, K1-5, and K1-4 with a fragment of K-5 [31]. Studies have shown that mainly the first three kringle domains are active in angiogenic inhibition, though a fourth also exhibits anti-angiogenic effects [28]. 


\section{Angiogenic Switch}

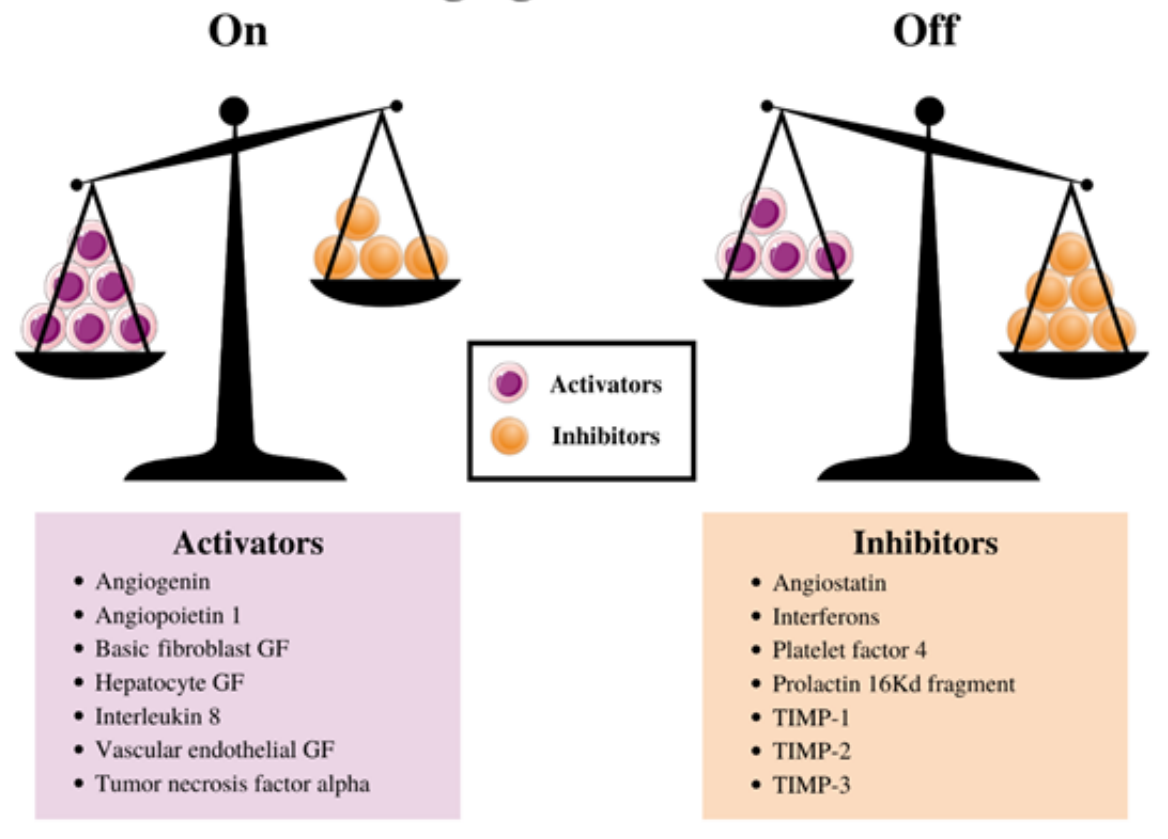

Figure 1: Angiogenesis scale.

Angiostatin displays a wide variety of functions, ranging from cell proliferation to apoptosis [32]. However, the primary role of angiostatin is to inhibit the proliferating endothelial cell compartment of newly vascularized blood vessels [33]. Much research has been done on the pathway for angiostatin to exhert anti-angiogenic effects, but the mechanism has not been identified. There are several potential mechanisms. One potential mechanism involves ATPase on the surface of endothelial cells because angiostatin can bind to mitochondrial F (1)-F (0) ATP synthase [27,34]. This ATP synthase likely plays a significant role in maintaining the intracellular $\mathrm{pH}$, while angiostatin inhibits the proton pump, acidifying the cell $[27,35]$. Pizzo et al. $[34,35]$ have speculated that this causes apoptosis in the endothelial cells. In a different study, Tarui et al. [36,37] discovered a different mechanism, where angiostatin binds to the $\alpha v \beta 3$-integrin on the surface of Chinese hamster ovary ( $\mathrm{CHO}$ ) cells and bovine arterial endothelial (BAE) cells. They also showed that plasmin is also a ligand to $\alpha v \beta 3$-integrin through binding to kringle domains and induces cell division [37] On the other hand, angiostatin does not induce cell division or cell migration because it is a known inhibitor of $\alpha v \beta 3$-integrin [37]. Although $\alpha v \beta 3$-integrin is an angiogenic activator, it is not required for angiogenesis because tissues without this factor can still undergo angiogenesis [37].

A third potential mechanism of angiogenic inhibition is via binding to angiomotin. Angiomotin consists of PDZ binding domains and conserved coiled coil [37]. Angiomotin binds to angiostatin on the endothelial cells, but it does not seem to be a surface receptor [38]. Nevertheless, angiomotin activates angiostatin, causing focal adhesion kinase (FAK) activity [28]. FAK plays a significant role in cell motility and substrate adhesion-dependent cell survival [39]. It may be plausible that FAK and angiostatin are both part of the same pathway that leads to apoptosis in endothelial cells [40].

Angiostatin is regulated by several molecules, the most prominent including neural/glial antigen 2 (NG2) and matrix metalloproteinases (MMPs) [41-43]. NG2 is an integral membrane proteoglycan found in rats, with the human homolog is chondroitin sulfate proteoglycan 4 (CSPG4) [44]. Soluble NG2 inhibits the antiangiogenic effect of angiostatin by binding to it [41]. MMPs are protease enzymes whose catalytic mechanism involves a metal, specifically calcium-dependent zinc-containing endopeptidases [45]. These enzymes primarily degrade extracellular matrix proteins. A study conducted by O'Reilly et al. [42] discovered that MMP-2, also known as gelatinase A, can produce angiostatin through processing plasminogen. Other studies have shown that MMP-2 has a similar role in the processing of other regulators such as TNF- $\alpha$ and TGF- $\alpha$ [42]. A different study by Chung et al. [43] showed that MMP-7, MMP-9, and MMP-12 also proteolytically cleave plasminogen into angiostatin, though MMP-9 is produced by endothelial cells while MMP-7 and MMP-12 are secreted by macrophages. MMP-2 and MMP-9 are activated in oxidant stress and when there is limited nitric oxide (NO) availability [43].

\section{Angiogenic Inhibitor Endostatin}

Endostatin is another key anti-angiogenic agent. Similar to other angiogenic inhibitors, endostatin is also generated from larger proteins. It is a $20 \mathrm{kDA}$ C-terminal fragment that is produced 
from the proteolytic cleavage of type XVIII collagen [46]. Type XVIII collagen can be found as a part of epithelial and endothelial basal membranes. Human endostatin consists of a zinc binding domain at the N-terminus, formed from His1, His3, His11, and Asp76. Ding et al. [47] suggest that the location of the zinc site at the $\mathrm{N}$-terminus right before the cleavage site may indicate the zinc is involved in anti-angiogenic activity, either in the cleavage process or immediately following cleavage [48].

Endostatin has been shown to block proliferation of endothelial cells into newly vascularized blood vessels in in-vitro studies [48]. In-vivo studies have shown that endostatin inhibited angiogenesis and growth of primary tumors and metastasis [46]. Currently, the mechanism of action still remains unclear, but recent studies have shown several possible pathways. One such pathway involves endostatin interfering with fibroblast growth factor-2 (FGF-2)induced signal transduction, which blocks endothelial cell motility and causes a variety of other effects, including blocking tumor necrosis factor (TNF)-induced activation of c-Jun NH2-terminal kinase (JNK)-dependent angiogenic activator gene expression and blocking signaling pathway between VEGF and VEGFR-1 $[40,46]$. Endostatin downregulates several signaling pathways associated with pro-angiogenic activity while simultaneously upregulating signaling pathways associated with anti-angiogenic activity [49].

\section{Therapeutic Potential of Angiogenesis Activators and} Inhibitors

Given that cancer angiogenesis is reliant on these angiogenic activators and inhibitors, it is acceptable to perceive the switch to an angiogenic state as an equilibrium shift between the positive regulators, the activators, and negative regulators, the inhibitors. Although angiogenesis is vital in cancer growth, inhibiting angiogenesis alone is rarely effective for most cancer patients [49]. Several drug treatments that regulate VEGF, angiostatin, and endostatin are currently in clinical trial or are being prescribed to patients. However, currently most anti-angiogenic drugs contain an anti-VEGF component as the primary target [49]. Some tumors may induce angiogenesis through other angiogenic factors, so it is vital to continue looking for other treatments.

Avastin, or bevacizumab, is a monoclonal antibody that inhibits angiogenesis by binding to VEGF-A, preventing it from binding to VEGFR-1 and -2. Bevacizumab was originally isolated from a mouse monoclonal antibody from mice immunized with VEGF165. The antibody was humanized by substituting several light and heavy chains, and the resulting plasmid was transferred to $\mathrm{CHO}$ cells to be produced at an industrial scale. Bevacizumab is given by slow injection into a vein and is used for glioblastomas and cancers of the kidney, lung, colon, rectum, ovary, cervix, and fallopian tube. It has shown to be effective in colorectal cancer treatment in combination with other drugs [50]. Bevacizumab was approved for metastatic colorectal cancer treatment by the FDA in 2004, and is generally effective as first-line therapy, usually in combination with chemotherapy or other treatments that enhance its efficacy. It has also shown promise in combination regimens for other cancers such as breast cancer and renal cell carcinoma. The recommended dose is $5 \mathrm{mg}$ per $\mathrm{kg}$ of body mass [51]. Some common side effects include hypertension, headache, hemorrhage, and wound healing complications [51].

Research on angiostatin is focusing on kringle domains. Many phase 1 clinical trials include recombinant angiostatin K1-3 [52]. A study by Sacco et al. showed that liposome-delivered angiostatin inhibits tumor growth in a transgenic model of spontaneous breast cancer [53]. Similarly, research on endostatin has gone through several stages of clinical trials as well. In one phase II clinical trial, 42 patients with pancreatic cancer were treated [54]. None of the patients who could be evaluated for a radiologic response experienced partial response to the therapy, meaning the treatment did not result in significant anti-tumor effects [54]. Other clinical trials testing endostatin in phase I clinical trials also show minimal anti-tumor effects compared to animal studies and pre-clinical studies. However, some positive therapeutic effect was observed, as certain cancer types exhibited partial regression and stabilization. ABT-510, a competitor with TSP-1 in CD36 binding, has been shown to stabilize certain cancer and cause partial tumor regression, and is now in phase II clinical trials.

Therapy using antiangiogenic agents typically produces mild side effects and is less toxic than other treatments because it is more specific-targeting angiogenic stimulators and angiogenic endothelial cells-and thus may be administered for longer periods. The process of anti-angiogenesis occurs by blocking tumor growth and metastasis, which effectively deprives the tumor of the nutrients it needs to grow. The aforementioned process of providing nutrients to developing cells is known as angiogenesis. They may significantly enhance other traditional cancer therapies and are being further studied as targets for cancer treatment. Currently, there are 14 approved therapy treatments involving anti-angiogenesis, and this number may rise in the future if studies continue. While not much is understood about these methods yet, they seem to bypass the harmful side effects that chemotherapy seems to entail [55].

\section{Metastatic Cascade}

Cancer metastasis, the spread of cancer which significantly increases disease risk, is a complex process usually characterized by three parts: invasion, intravasion, and extravasion. While primary cancer itself can be deadly, metastasis is responsible for the higher mortality rates in patients due to the spread of the disease that it causes. Tumor cells that are able to penetrate the surrounding tissues have the ability to invade the extracellular matrix, traveling throughout the body via the bloodstream or lymph system and gaining access to non-adjacent body parts. Once they find an area to attach themselves to, they will proliferate and grow a second tumor at that location. Understanding this process in more detail is the key to preventing the metastasis of cancer in future patients, thereby reducing the risk of death [2]. To put it into further detail, this complex cascade involves tumor cells invading the extracellular matrix, translocating to distant tissues, and surviving, proliferating again, and thriving in the distant environments as 
well as the original. The invasion begins the process when cancer cells breach the extracellular matrix, allowing entry into the stroma. During intravasation, these cancerous cells are able to spread lymphatically; thus, this is largely dependent on angiogenic promoters such as VEGFs. These tumor cells must survive stresses such as lack of anchorage to the matrix and mechanisms of the innate immune system, especially natural killer cells, and arrive at distant sites which allow adhesion and proliferation. This begins the process of extravasion, which is dependent on the cancerous cells' ability to survive and thrive in the foreign microenvironment, forming metastases [56].

\section{Role of Oncogenic Transcription Factors in Cancer Metastasis}

Tumor progression affecting cancer metastasis is heavily reliant on gene regulation and epigenetic modifications. Oncogenic transcription factors such as c-Myc represent classes of oncogenes that increase metastasis. Myc functions in both the primary formation of tumors as well as metastasis and is linked to the epithelial-mesenchymal transition, which allows further spread of cancer cells. Others, such as Kruppel-like factor 4 (KLF4), act as metastasis suppressors because they act as tumor suppressors [57]. One key oncogenic transcription factor is an inactivated or mutant p53 gene. Homologs of this gene, p73 and p63, have also been characterized to have similar functions, although they are rarer in human tumors. Mutant proteins resulting from the p53 gene are vastly due to missense mutations, and are abundant in malignancies of the lungs, colon, and breast. Although more research needs to be done to fully understand its molecular bases, the mutant proteins have a prolonged half-life, likely due to inefficient degradation; thus, they are much more unstable in normal tissues. Findings from in vitro studies have shown that new oncogenic functions are found in these proteins, causing the tumor to spread and become resistant to treatment [58].

E26 transformation specific (ETS) related genes (ERGs) also play significant roles in cancer metastasis, along with roles in inflammation, migration, and invasion alongside angiogenesis [59]. Chromosomal translocation and amplification cause increased ETS signaling, which enhances tumorigenesis [60]. In the specific case of ERG, its activation and overexpression are often associated with prostate cancer because it inactivates phosphatase and tensin homologue, a tumor suppressor gene [59].

\section{Conclusion}

Angiogenesis is a crucial part of metastasis, and transcription factors are significant players in both processes. For tumors to reach metastatic potential, they must switch from avascular to vascular by upregulating pro-angiogenic factors such as VEGF and downregulating anti-angiogenic factors such as angiostatin and endostatin [61]. The complex pathway leading to cancer is, thus, dependent on these transcription factors and angiogenic activators and inhibitors. Because of this, they are heavily considered as potential targets for treatment in the future [62].

\section{Conflicts of Interest}

None to declare.

\section{Acknowledgment}

We thank Ava Wang for generating the figures used in this paper.

\section{References}

1. Sever R, Brugge JS (2015) Signal transduction in cancer. Cold Spring Harb Perspective Med 5(4): a006098.

2. Martin TA, Ye L, Sanders AJ, Lane J, Jiang WG (2013) Cancer invasion and metastasis: Molecular and cellular perspective.

3. Thiery JP (2002) Epithelial-mesenchymal transitions in tumor progression. Nat Rev Cancer 2(6): 442-454.

4. Seyfried TN, Huysentruyi TN (2013) On the origin of cancer metastasis. Crit Rev Oncog 18(1-2): 43-73.

5. Bhagwat AS, Vakoc CR (2015) Targeting transcription factors in cancer. Trends Cancer 1(1): 53-65.

6. Cooper JA. Transcription factor. Biology.

7. Oettgen P (2010) The role of ets factors in tumor angiogenesis. J Oncol 2010: 767384 .

8. Zheng H, Wasylyk C, Ayadi A, Abecassis J, Schalken JA, et al. (2003) The transcription factor net regulates the angiogenic switch. Genes Dev 17(18): 2283-2297.

9. Sartippour MR, Shao ZM, Heber D, Beatty P, Zhang L, et al. (2002) Green tea inhibits vascular endothelial growth factor (VEGF) induction in human breast cancer cells. Nutr J 132(8): 2307-2311.

10. Nishida N, Yano H, Nishida T, Kamura T, Kojiro M (2006) Angiogenesis in cancer. Vasc Health Risk Manag 2(3): 213-219.

11. Muthukkaruppan VR, Kubai L, Auerbach R (1982) Tumor-induced neovascularization in the mouse eye. J Natl Cancer Inst 69(3): 699-708.

12. Gille J (2006) Antiangiogenic cancer therapies get their act together: Current developments and future prospects of growth factor- and growth factor receptor-targeted approaches. Exp Dermatol 15(3): 175186.

13. Holmogren L, O’Reilly MS, Folkman J (1995) Dormancy of micrometastases: Balanced proliferation and apoptosis in the presence of angiogenesis suppression. Nat Med 1(2): 149-153.

14. Khosravi-Shahi P, Castillo-Rueda AD, Perez-Manga G (2008) Neoplastic angiogenesis. An Med Interma 25(7): 366-369.

15. Lee SH, Jeong DJ, Han YS, Baek MJ (2015) Pivotal role of vascular endothelial growth factor pathway in tumor angiogenesis. Ann Surg Treat Res 89(1): 1-8.

16. Rao N, Lee YF, Ge R (2015) Novel endogenous angiogenesis inhibitors and their therapeutic potential. Acta Pharmacol Sin 36(10): 1177-1190.

17. Xiang W, Ke Z, Zhang Y, Cheng GH, Irwan ID, et al. (2011) Isthmin is a novel secreted angiogenesis that inhibits tumor growth in mice. J Cell Mol Med 15(2): 359-374.

18. Niu G, Chen X (2010) Vascular endothelial growth factor as an antiangiogenic target for cancer therapy. Curr Drug Targets 11(8): 10001017.

19. Kazemi M, Carrer A, Moimas S, Zandona L, Bussani R, et al. (2016) VEGF121 and VEGF165 differentially promote vessel maturation and tumor growth in mice and humans. Cancer Gene Ther 23(5): 125-132.

20. Lenz HJ (2005) Antiangiogenic agents in cancer therapy. Oncol 19(4 Suppl 3): 17-25. 
21. Maxwell PH, Ratcliffe PJ (2002) Oxygen sensors and angiogenesis. Semin Cell Dev Biol 13(1): 29-37.

22. Bosco MC, D’Orazi G, Del Bufalo D (2020) Targeting hypoxia in tumor: a new promising therapeutic strategy. J Exp Clin Cancer Res 39(1): 8.

23. Gordan JD, Simon MC (2007) Hypoxia-inducible factors: central regulators of the tumor phenotype. Curr Opin Genet Dev 17(1): 71-77.

24. Kim H, Ko Y, Park H, Zhang H, Jeong Y, et al. (2019) MicroRNA-148a/b$3 p$ regulates angiogenesis by targeting neuropilin-1 in endothelial cells. Exp Mol Med 51(11): 134.

25. Ding Z, ZHu J, Zeng Y, Du W, Zhang Y, et al. (2019) The regulation of neuropilin 1 expression by miR-338-3p promotes non-small cell lung cancer via changes in EGFR signaling. Mol Carninog 58(6): 1019-1032.

26. Yan XC, Cao J, Liang L, Wang L, Gao F, et al. (2016) miR-342-5p is a notch downstrean molecule and regulates multiple angiogenic pathways including notch, vascular endothelial growth factor and transforming growth factor $\beta$ signaling. J Am Heart Assoc 5(2).

27. Pandya NM, Dhalla NS, Santani DD (2006) Angiogenesis-a new target for future therapy. Vascul Pharmacol 44(5): 265-274.

28. Geiger JH, Cnudde SE (2004) What the structure of angiostatin may tell us about its mechanism of action. J Thromb Haemost 2(1): 23-24.

29. Cao Y, Ji RW, Davidson D, Schaller J, Marti D, et al. (1995) Kringle domains of human angiostatin. Characterization of the anti-proliferative activity on endothelial cells. J Biol Chem 271(46): 29461-29467.

30. O’Reilly MS, Holmgren L, Shing Y, Chen C, Rosenthal RA, et al. (1994) Angiostatin: A novel angiogenesis inhibitor that mediates the suppression of metastases by a lewis lung carcinoma. Cell 79(2): 315328.

31. Abad MC, Arni RK, Grella DK, Castellino FJ, Tulinksy A, et al. (2002) The $\mathrm{x}$-ray crystallographic structure of the angiogenesis inhibitor angiostatin. J Mol Biol 318(4): 1009-1017.

32. Claesson-Welsh L, Welsh M, Ito N, Anand-Apte B, Soker S, et al. (1998) Angiostatin induces endothelial cell apoptosis and activation of focal adhesion kinase independently of the integrin-binding motif RGD. Proc Natl Acad Sci USA 95(10): 5579-5583.

33. Cao Y (2001) Endogenous angiogenesis inhibitors and their therapeutic implications. Int J Biochem Cell Biol 33(4): 357-369.

34. Moser TL, Stack MS, Asplin I, Enghild JJ, Pizzo SV, et al. (1999) Angiostatin binds ATP synthase on the surface of human endothelial cells. Proc Natl Acad Sci USA 96(6): 2811-2816.

35. Moser TL, Kenan DJ, Ashley TA, Roy JA, Goodman MD, et al. (2001) Endothelial cell surface F1-F0 ATP synthase is active in ATP synthesis and is inhibited by angiostatin. Proc Natl Acad Sci USA 98(12): 66566561.

36. Tarui T, Miles LA, Takada Y (2001) Specific interaction of angiostatin with integrin alpha(v) beta (3) in endothelial cells. J Biol Chem 276(43): 39562-39568.

37. Tarui T, Majumdar M, Miles LA, Takada Y (2002) Plasmin-induced migration of endothelial cells. A potential target for the anti-angiogenic action of angiostatin. J Biol Chem 277(37): 33564-33570.

38. Troyanovsky B, Levchenko T, Mansson G, Matvijenko O, Holmgren L (2001) Angiomotin: An angiostatin binding protein that regulates endothelial cell migration and tube formation. J Cell Biol 152(6): 12471254 .

39. Plopper GE, McNamee HP, Dike LE, Bojanowski K, Ingber DE (1995) Convergence of integrin and growth factor receptor signaling pathways within the focal adhesion complex. Mol Biol Cell 6(10): 1349-1365.

40. Nyberg P, Xie L, Kalluri R (2005) Endogenous inhibitors of angiogenesis. Cancer Res 65(10): 3967-3979.
41. Wahl ML, Kenan DJ, Gonzalez-Gronow M, Pizzo SV (2005) Angiostatin's molecular mechanism: aspects of specificity and regulation elucidated. J Cell Biochem 96(2): 242-261.

42. O’Reilly MS, Wiederschain D, Stetler-Stevenson WG, Folkman J, Moses MA (1999) Regulation of angiostatin production by matrix metalloproteinase-2 in a model of concomitant resistance. J Biol Chem 274(41): 29568-29571.

43. Chung AWY, Hsiang YN, Matzke LA, McManus BM, van Breemen C, et al. (2006) Reduced expression of vascular endothelial growth factor paralleled with the increased angiostatin expression resulting from the upregulation activities of matrix metalloproteinase- 2 and -9 in human type 2 diabetic arterial vasculature. Circ Res 99: 140-148.

44. Asher RA, Morgenstern DA, Moon LD, Fawcett JW (2001) Chondroitin sulfate proteoglycans: Inhibitory components of the glial scar. Prog Brain Res 132: 611-619.

45. Verma RP, Hansch C (2007) Matrix metalloproteinases (MMPs): Chemical-biological functions and (Q)SARs. Bioorg Med Chem 15(6): 2223-2268.

46. O’Reilly MS, Boehm T, Shing Y, Fukai N, Vasios G, et al. (1997) Endostatin: An endogenous inhibitor of angiogenesis and tumor growth. Cell 88(2): 277-285.

47. Ding YH, Javaherian K, Lo KM, Chopra R, Boehm T, et al. (1998) Zincdependent dimers observed in crystals of human endostatin. Proc Natl Acad Sci USA 95(18): 10443-10448.

48. Folkman J, Kalluri R (2004) Cancer without disease. Nature 427(6977): 787.

49. Cao Y (2016) Future options of anti-angiogenic cancer therapy. Chin J Cancer 35: 21.

50. Sund M, Zeisberg M, Kalluri R (2005) Endogenous stimulators and inhibitors of angiogenesis in gastrointestinal cancers: Basic science to clinical applications. Gastroenterol 129(6): 2076-2091.

51. Shih T, Lindley C (2006) Bevacizumab: An angiogenesis inhibitor for the treatment of solid malignancies. Clin Ther 28(11): 1779-1802.

52. Soff GA (2000) Angiostatin and angiostatin-related proteins. Cancer Metastasis Rev 19(1-2): 97-107.

53. Sacco MG, Caniatti M, Cato EM, Frattini A, Chiesa G, et al. (2000) Liposome-delivered angiostatin strongly inhibits tumor growth and metastatization in a transgenic model of spontaneous breast cancer. Cancer Res 60(10): 2660-2665.

54. Kulke MH, Bergsland EK, Ryan DP, Enzinger PC, Lynch TJ, et al. (2006) Phase II study of recombinant human endostatin in patients with advanced neuroendocrine tumors. J Clin Oncol 24(22): 3555-3561.

55. Feng X, Ofstad W, Hawkins D (2010) Antiangiogenic therapy: A new strategy for cancer treatment. US Pharm 35(7): 4-9.

56. Valastyan S, Weinberg RA (2011) Tumor metastasis: Molecular insights and evolving paradigms. Cell 147(2): 275-292.

57. Ell B, Kang Y (2013) Transcriptional control of cancer metastasis. Trends Cell Biol 23(12): 603-611.

58. Strano S, Dell'Orso S, Di Agnostino S, Fontemaggi G, Sacchi A, et al. (2007) Mutant p53: An oncogenic transcription factor. Oncogene 26(15): 22122219.

59. Adamo P, Porazinski S, Rajatileka S, Jumbe S, Hagen R, et al. (2017) The oncogenic transcription factor ERG represses the transcription of the tumor suppressor gene PTEN in prostate cancer cells. Oncol Lett 14(5): 5605-5610.

60. Sizemore GM, Pitarresi JR, Balakrishnan S, Ostrowski MC (2017) The ETS family of oncogenic transcription factors in solid tumors. Nat Rev Cancer 17(6): 337-351. 
61. Folkman J (2002) Role of angiogenesis in tumor growth and metastasis. Semin Oncol 29(6 Suppl 16): 15-18.
62. Darnell JE (2002) Transcription factors as targets for cancer therapy. Nat Rev Cancer 2(10): 740-749. 